\title{
Computational Simulation of Carotid Artery: From Patient-Specific Images to Finite Element Analysis
}

\author{
S. Djorovic ${ }^{1,2^{*}}$, I. Saveljic ${ }^{1,2}$, N. Filipovic ${ }^{1,2}$ \\ ${ }^{1}$ Faculty of Engineering, University of Kragujevac, 6 Sestre Janjic Street, 34000 Kragujevac, \\ Serbia \\ ${ }^{2}$ Research and Development Center for Bioengineering (BioIRC), 6 Prvoslava Stojanovica \\ Street, 34000 Kragujevac, Serbia \\ e-mails: smiljana@kg.ac.rs, isaveljic@kg.ac.rs, fica@kg.ac.rs \\ *corresponding author
}

\begin{abstract}
Nowadays, finite element analysis is a well-assessed technique which enables investigation of blood vessels behavior under different boundary conditions. Given the rapid progression of both medical imaging techniques and computational methods, the challenge of using the simulation of human arteries such as carotid arteries to address different medical conditions and support the clinical practice can be approached. Within this context, this study investigates the recent achievements in the field of computational examinations of carotid artery and presents the method for analysis of patient-specific carotid artery model and its application for simulation of atherosclerosis progression. In particular, we focus on the patient-specific anatomical geometry reconstruction and then on the examination of the plaque progression within carotid artery, by examining the parameters such as blood velocity and shear stress distribution. This type of simulation and determination of plaque zone and its progression in time for a specific patient has shown a potential benefit for future prediction of this vascular disease using the computer simulation.
\end{abstract}

Keywords: Carotid artery, computational model, plaque progression, finite element method, numerical simulation

\section{Introduction}

Carotid arteries are major blood vessels on the right and left side of the neck that supply oxygenated blood to the brain, face and neck. Carotid artery starts from Common Carotid Artery (CCA) and branches into Internal Carotid Artery (ICA - supplies blood to the brain) and External Carotid Artery (ECA - supplies blood to the face and neck). Carotid artery role in cerebrovascular system is of vital importance and, therefore, its malfunctions and diseases have been considered and examined by experts in different fields (medical scientists, biochemists and bioengineers). One of the most prevalent carotid artery diseases is atherosclerosis, which presents lumen occlusion due to building up of plaque within the arterial wall. This is the inflammatory disease caused by cholesterol deposits and cellular proliferation. Untreated carotid atherosclerosis disease 
alters blood flow, promotes thrombus and embolus formation that lead to ischemic stroke. Approximately $10 \%$ of ischemic strokes in the world are caused by carotid atherosclerosis solely (Chaturvedi \& Sacco, 2015).

In the atherosclerosis diagnostic process, arterial wall properties, plaque composition and hemodynamical parameters can precisely determine the progression of disease. With that aim, computational modelling of atherosclerosis has been performed in recent years (Parton et al. 2016). Also, the relation between atherosclerosis progression and existence of blood recirculation zone, low wall shear stress and high oscillating shear index within the carotid arteries have been studied (Sousa et al. 2016). The mechanisms of atherosclerosis have been used to provide insights for understanding the processes which lead to plaque initiation and development, as well as to predict plaque regions and mechanisms which are prone to disease progression (Filipovic et al. 2013; Bourantas et al. 2016).

It is found that wall shear stress distribution and Low-Density Lipoprotein (LDL) transport are associated with plaque progression. Researchers have added mass transfer equation to the calculations and studied concentration of LDL particles within cardiovascular system. For example, the research of Nematollahi et al. (2012) indicated that LDL surface concentration increases in regions associated with high infiltration rate (high blood pressure) and low wall shear stress. Sakellarios et al. (2017) simulated atherosclerotic progression using LDL transport modelling and serial CT coronary angiographic study. They also performed multivariate analysis of the variables (lumen area, plaque area, plaque burden and necrotic core) associated with atherosclerosis progression.

In addition, examining how atherosclerotic plaque forms depends on mechanical properties and reaction with arterial wall is challenging and can be implemented to discover plaque rupture mechanism. Due to complex interaction between arterial wall and blood, many researchers have implemented Fluid-Solid Interaction (FSI) analysis in their numerical investigations of carotid atherosclerosis disease (Lee et al. 2004; Xiaojuan et al. 2015; Valencia and Baeza 2009). These types of studies need the usage of acquired data from diagnostic tools such as Computed Tomography (CT) scan, Ultrasound (US), Magnetic Resonance Imaging (MRI), angiography, Laser Doppler Anemometer (LDA), Power Doppler Imaging (PDI), Doppler Sonography (DS), spectroscopy and other conventional pathological tests.

In novel approach, Tomaso et al. (2015) combined experimental and numerical methods of biomechanical and biochemical atherosclerosis progression modeling and introduced a promising outline for determination of patient-specific plaque growth simulation. Although researchers have simulated different aspects of carotid atherosclerosis disease, it is obvious that models which consider biochemical and biomechanical aspects of atherosclerosis progression are more accurate.

Based on previous investigations, the main aim of this study was to computationally simulate plaque progression within right carotid artery, considering patient-specific geometry and biomolecular parameters. The CT scan images were used for creation of the 3D patient-specific model. The parameters such as velocity and shear stress distribution were determined in two time periods (1. baseline and 2. follow-up) using Finite Element Method (FEM) approach.

The rest of the paper is organized as follows. Section 2 describes methods used for creation of the patient-specific carotid artery model and simulation of atherosclerotic plaque progression. The types of the data (US, MRI, CT) which can be used for the 3D geometrical reconstruction are summarized. Procedure for creation of the 3D patient-specific model which required use of several software packages is presented in Section 2.1. Section 2.2 describes general concept of the finite element analysis for simulation of atherosclerotic plaque progression (blood flow and mass transport). The governing equations employed in the simulation and based on the FEM are 
given in Section 2.3. It should be noted that the PAK in-house solver was employed for performing the computational simulation. Section 3 covers discussion of results (blood velocity and shear stress distribution) given for atherosclerotic baseline and follow-up of atherosclerosis progression. Finally, Section 4 presents the main conclusions of this study and plans for further improvements.

\section{Materials and Methods}

This section presents the type of data which can be used for reconstruction of the 3D geometry of the human carotid artery and describes the procedure for creation of the $3 \mathrm{D}$ patient-specific model of right carotid artery used in the simulation of atherosclerotic plaque progression. In recent years, the $3 \mathrm{D}$ reconstruction of the blood vessels has been widely used as a way to determine the correct diagnosis of disease. Namely, accurate characterization of carotid artery geometry is vital for our understanding of the atherosclerosis pathogenesis (Kamenskiy et al. 2012). Therefore, patient-specific models are necessary in order to take into consideration the individual anatomy of the patient. During the reconstruction of carotid artery geometry, different data acquired from diagnostic procedures can be used, such as US, MRI, or CT scan. The US imaging has been used to measure wall thickness, blood velocities, and identify the patients with a histologically unstable carotid plaque (Salem et al. 2014). Furthermore, the US imaging can be used for automated lumen and adventitial border delineation of carotid artery wall (Kumar et al. 2018). Compared with the US, the CT and MRI are widely used for reconstruction of patientspecific geometries (Antiga at al. 2003; Milašinović et al. 2008; Moussawi et al. 2014). Besides these methods, LDA, PDI, DS, angiography, spectroscopy, and other conventional pathological tests can be used in the analysis of atherosclerosis progression.

In this paper, the CT scan images were used to create the geometry which included CCA, ICA and ECA. Reconstruction and modeling were carried out in several stages with the aim to create a real anatomical 3D geometry. To accomplish this, several different software packages were used, whose various tools and functions contributed to the optimization of surface elements, 3D elements, and preparation of the model for the blood flow simulation coupled with the mass transfer and diffusion within the blood vessel wall (Section 2.1). In addition, the computational simulation of the atherosclerotic plaque progression and boundary conditions are described (Section 2.2). Finally, the governing equations employed in the simulation and based on the FEM are presented (Section 2.3)

\subsection{Creation of patient-specific model}

The whole process of the $3 \mathrm{D}$ reconstruction of the blood vessels can be divided into three steps:

1. Segmentation of 2D image,

2. Smoothing the blood vessel,

3. $3 \mathrm{D}$ reconstruction of blood vessels.

In order to perform the computational analysis of plaque progression within the carotid artery, the patient-specific 3D model was created. Geometry of the lumen domain was reconstructed using the CT scan images. The set of DICOM images, with resolution of $512 \times 512$ $\mathrm{mm}$ were imported in segmentation software, able to calculate $3 \mathrm{D}$ model from $2 \mathrm{D}$ images. In order to reconstruct the region of interest, each image was segmented using the manipulation in three different planes (Fig. 1), which enabled the creation of more accurate 3D model of the patient's anatomy. 

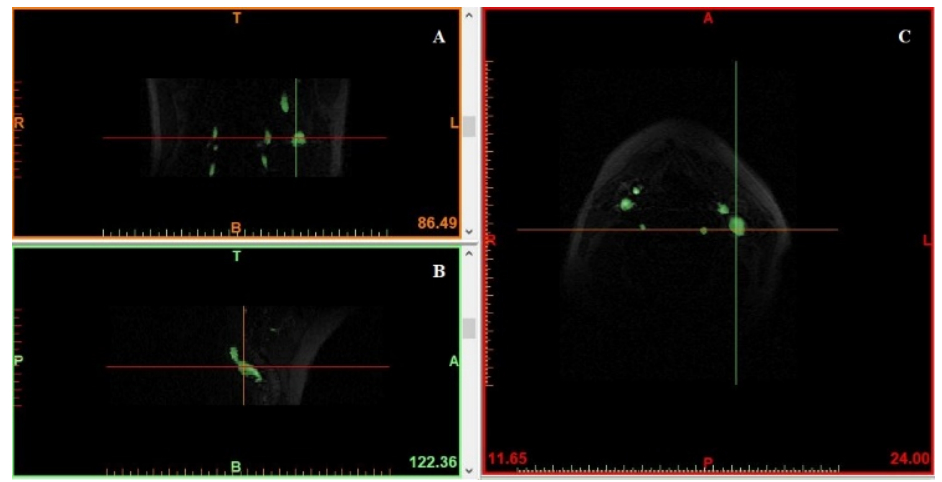

Fig. 1. Coronal (A), sagittal (B) and transversal (C) cross-section of the modeled carotid artery.

The region of interest selected in the segmentation process is converted to the $3 \mathrm{D}$ surface model using an adapted algorithm. The stereolithography (STL) representation set the boundary surface for the lumen. After obtaining the initial 3D model, optimization of the surface mesh was performed in Geomagic software, in order to create mesh suitable for the computational simulation (Fig. 2). This software focuses on reconstruction of 3D surface model and its real representation which is widely used in different fields of bioengineering. The difference between the CCA, ECA and ICA can be seen in Fig. 2. Furthermore, the patient whose CT scan images were used had the mild stenosis at the ICA, which is common among patients since atherosclerosis symptoms can be usually observed after the formation of stenosis.

After reconstruction of the carotid artery, the surface model was exported in the STL format from Geomagic to Femap software. Femap was used in combination with our in-house software for the conversion of tetrahedral elements to eight-node brick elements. The dataset was imported into the PAK in-house solver for further simulation of blood flow and mass transport through the carotid artery (Kojic et al. 2008).

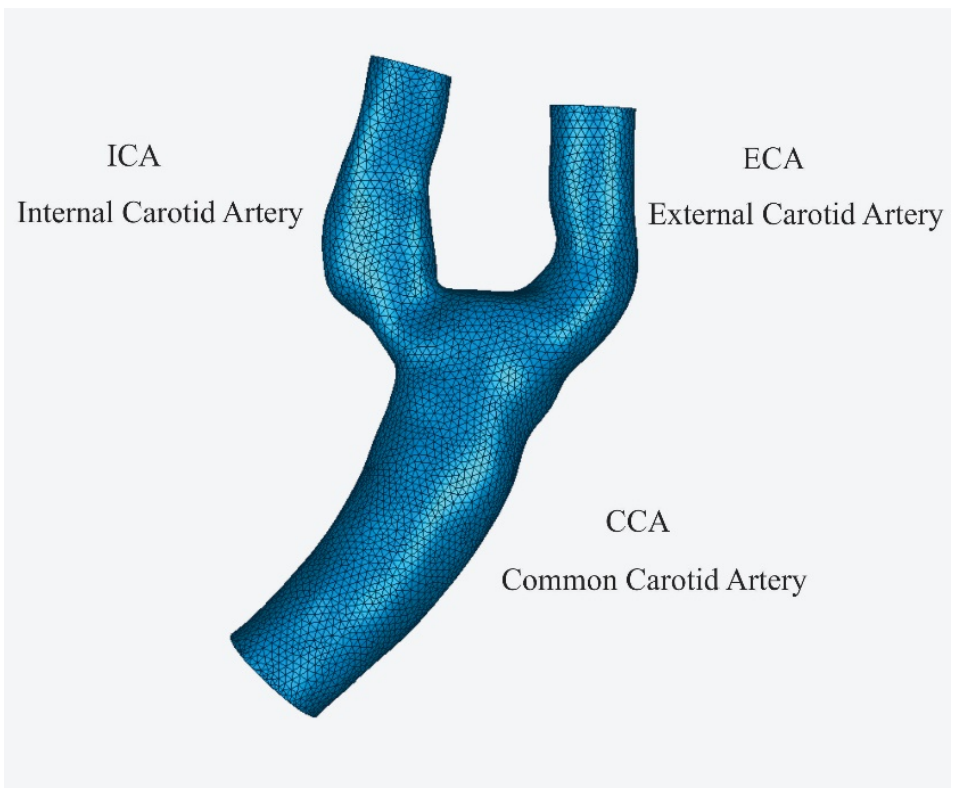

Fig. 2. 3D finite element mesh of carotid artery (baseline). 


\subsection{Numerical analysis}

In the presented study, the LDL mass transfer through the blood lumen and arterial wall was governed by different sets of equations in order to computationally simulate atherosclerosis progression for the specific patient. The transport of LDL in the lumen of the carotid artery and through its arterial tissue were coupled by Kedem-Katchalsky equations. The mass transfer in the lumen was modeled using convective diffusion equations, while convective diffusion reactive equations were used for modeling mass transfer in the arterial wall. The fluid filtration (mass transfer across the wall of the blood vessel) was modelled with the Darcy's law. The NavierStokes equations and continuity equation were applied for the simulation of blood flow in the lumen. The biomolecular parameters and adhesion molecules were also used for computer simulation. These methods are explained in detail by Filipovic et al. (2013). Also, the 3D meshmoving Arbitrary Lagrangian-Eulerian (ALE) formulation was applied to follow the wall displacements and change of the carotid wall geometry during the plaque growth (Filipovic et al. 2006a)

The average blood properties such as dynamic viscosity of $0.00365 \mathrm{~Pa} \cdot \mathrm{s}$ and density of 1050 $\mathrm{kg} / \mathrm{m}^{3}$ were prescribed with the aim to perform analysis which reflects the realistic human blood flow. Also, the appropriate boundary conditions were employed, such as: prescribed blood velocity at the inlet of the arterial segment and physiological resistance pressure at the arterial outlets.

\subsection{Computer model}

The continuum-based approach for plaque formation and development in $3 \mathrm{D}$ is presented in this section. The blood flow was simulated by the 3D Navier-Stokes equations (1), together with the continuity equation (2):

$$
\begin{gathered}
-\mu \nabla^{2} u_{l}+\rho\left(u_{l} \cdot \nabla\right) u_{l}+\nabla p_{l}=0 \\
\nabla u_{l}=0
\end{gathered}
$$

where $u_{l}$ is blood velocity, $p_{l}$ is pressure, $\rho$ is density and $\mu$ is blood dynamic viscosity (Kojic et al. 2008; Filipovic et al. 2006b). Darcy's Law for Newtonian fluids was used to model mass transfer across the wall of the blood vessel, together with continuity equation:

$$
\begin{gathered}
u_{w}-\nabla\left(\frac{k}{\mu_{p}} p_{w}\right)=0, \\
\nabla u_{w}=0,
\end{gathered}
$$

where $u_{w}$ is transmural velocity, $k$ is the Darcian permeability coefficient of the arterial wall, $p_{w}$ is the pressure in the arterial wall and $\mu_{p}$ is viscosity of blood plasma.

Mass transfer in the lumen domain was modeled using convective diffusion equations(5):

$$
\nabla \cdot\left(D_{l} \nabla c_{l}+c_{l} u_{l}\right)=0
$$

where $D_{l}$ is diffusion coefficient of the lumen while the $c_{l}$ represents blood concentration. Convective diffusion reactive equations (6) were used for modeling mass transfer in the wall which was related to transmural flow:

$$
\begin{gathered}
\nabla \cdot\left(-D_{w} \nabla c_{w}+\mathrm{K} c_{w} u_{w}\right)=r_{w} c_{w}, \\
\nabla \cdot\left(-D_{w} \nabla c_{w}+\mathrm{K} c_{w} u_{w}\right)=r_{w} c_{w},
\end{gathered}
$$


where $D_{w}$ is diffusive coefficient of solution in the wall, $c_{w}$ is solute concentration, $\mathrm{K}$ is solute lag coefficient and $r_{w}$ is consumption rate constant.

LDL transport in the lumen of the vessel was coupled with Kedem-Katchalsky equations (7) and (8):

$$
\begin{gathered}
J_{v}=L_{p}\left(\Delta p-\sigma_{d} \Delta \pi\right), \\
J_{s}=P \Delta c+\left(1-\sigma_{f}\right) J_{v} \bar{c},
\end{gathered}
$$

where $L_{p}$ is the hydraulic conductivity of the endothelium, $\Delta p$ is the pressure drop across the endothelium, $\Delta \pi$ is the oncotic pressure difference across the endothelium, $\Delta c$ is the solute concentration difference across the endothelium, $\sigma_{d}$ is the osmotic reflection coefficient, $\sigma_{f}$ is the solvent reflection coefficient, $P$ is the solute endothelial permeability, and $\bar{c}$ is the mean endothelial concentration.

Three additional partial differential equations were used for solving the inflammatory process (Calvez et al. 2008):

$$
\begin{gathered}
\partial_{t} \mathrm{O} x=d_{2} \Delta \mathrm{O} x-k_{1} \mathrm{O} x \cdot M, \\
\partial_{t} \mathrm{M}+\operatorname{div}\left(v_{w} M\right)=d_{1} \Delta M-k_{1} \mathrm{O} x \cdot M+S /(1-S), \\
\partial_{t} S=d_{3} \Delta S-\lambda S+k_{1} \mathrm{O} x \cdot M+\gamma\left(O x-O x^{t h r}\right),
\end{gathered}
$$

where $\mathrm{O} x$ is the oxidized LDL transport or $c_{w}$ - the solute concentration in the wall from (6); $M$ and $S$ are concentrations in the intima of macrophages and cytokines, respectively; $d_{1}, d_{2}, d_{3}$ are the corresponding diffusion coefficients; $\lambda$ and $\gamma$ are degradation and LDL oxidized detection coefficients; and $v_{w}$ is the inflammatory velocity of plaque growth, which satisfies Darcy's law and continuity equation (3) (4) (Kojic et al. 2008; Filipovic et al 2016a; Filipovic et al. 2006b).

After prescribed boundary conditions and material characteristic, as well as different sets of equations for mass transfer modeling, the PAK in-house solver (Kojic et al. 2008) was used for the computational simulation. It calculated blood velocity and shear stress distribution within the patient-specific carotid artery, considering the baseline and follow-up time periods.

\section{Results and Discussion}

This study was based on modeling of the patient-specific carotid artery using the CT scan images in combination with several different software packages. The 3D blood flow and the LDL transport through the lumen and artery wall, coupled with plaque progression in the carotid artery wall were simulated based on FEM. The biomolecular parameters and adhesion molecules were prescribed for the computer simulation (baseline and follow-up). The appropriate boundary conditions were also prescribed. The 3D blood flow was governed by the Navier-Stokes equations, together with the continuity equation. Mass transfer within the blood lumen and through the arterial wall was coupled with the blood flow and is modelled by the convectiondiffusion equation. Kedem-Katchalsky equations described the LDL transport in lumen of the carotid artery and through the vessel tissue. The PAK in-house solver was employed for performing the computational simulation.

The results of plaque progression simulation are examined in two time points: 1 . baseline and 2. follow-up (Fig. 3 and Fig. 4, respectively), presenting the velocity vector field and shear stress distributions. Alterations of these parameters during the simulated plaque progression can 
be noticed by comparing Fig. 3 and Fig. 4. Low blood velocity (Fig. 3 a)) and shear stress (Fig. $3 \mathrm{~b})$ ) are present at the carotid bifurcation, which is a critical region for atherosclerosis development. Also, low values of blood velocity and shear stress are associated with increment in endothelial cells permeability and reactions, as well as plaque formation regions (Tomaso et al. 2015). The patient whose CT scan images were used for modelling of carotid artery geometry had mild stenosis at the ICA, which is also prone to the atherosclerotic plaque development due to sinusoidal enlargement (Fig. 3). Presence of mild stenosis is common among patients since atherosclerosis symptoms can be usually observed after the formation of such stenosis.
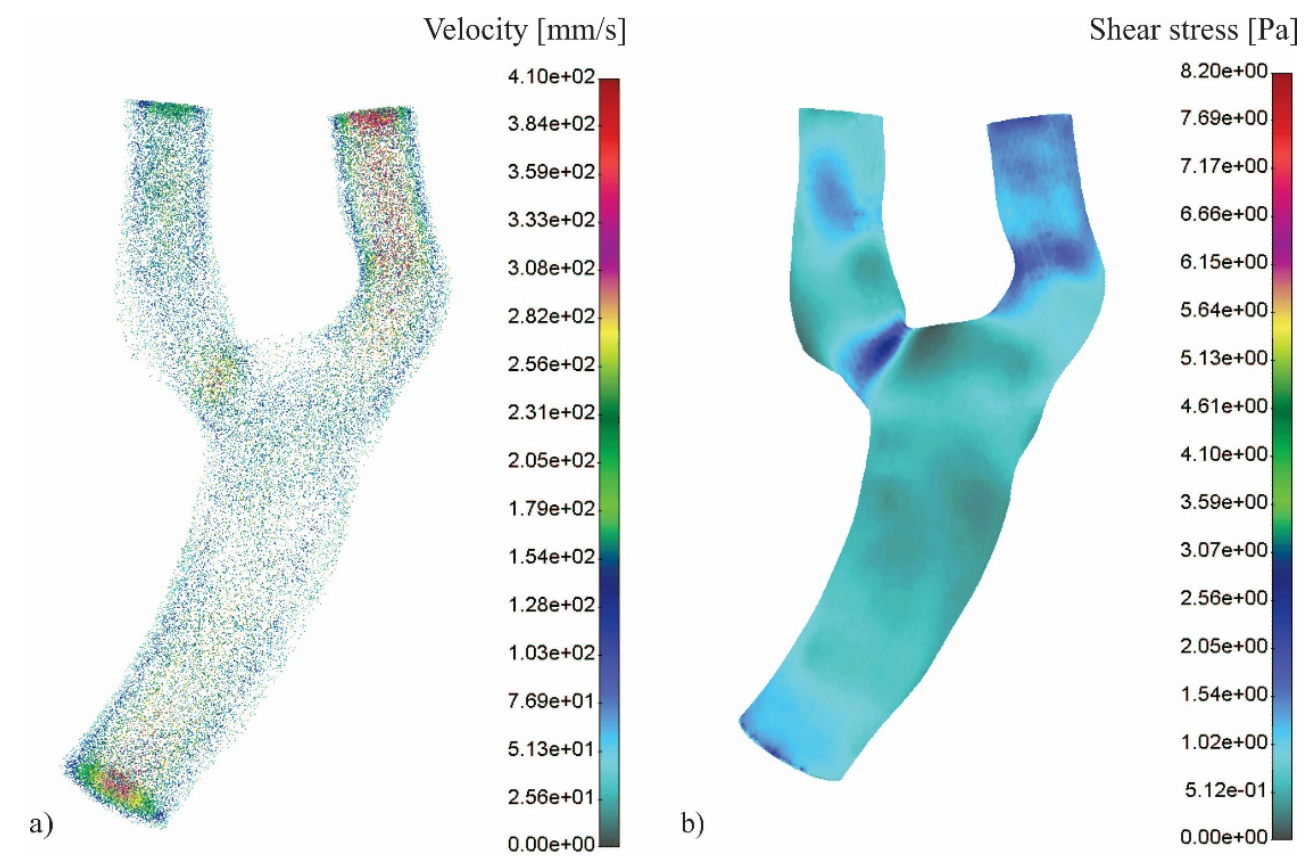

Fig. 3. Baseline: a) Velocity distribution vector field and b) Shear stress distribution.

Further development of atherosclerosis leads to increase of plaque volume and decrease of lumen diameter, which is followed by reduced blood flow through stenotic zone. Thus, after the follow-up a decreased diameter of the ICA can be seen (Fig. 4). The stenotic zone after the simulated plaque progression is matched with the mild zone from the baseline. Recirculation zone is still present at the carotid bifurcation, before the stenosis (Fig 4. a)), while the blood has increased velocity through the stenosis. The shear stress is increased at the stenotic zone (marked area) and the maximum value of the shear stress for the obtained geometry is $10.1 \mathrm{~Pa}$ (Fig $4 \mathrm{~b}$ )). On the other hand, low shear stress is present at the carotid bifurcation zone, which together with the presence of the recirculation zone indicates the possibility for further plaque progression.

Compared with baseline, the carotid diameter is approximately $35 \%$ reduced. During the atherosclerosis progression, the estimation of diameter reduction is significant for further steps in medical treatment. In addition, this type of simulation has an increasing importance in medicine with the aim to facilitate patient's monitoring and contribute to prognosis and decision making. Also, determination of plaque location for a specific patient, coupled with computer simulation of plaque progression in time, has a potential benefit for prediction of atherosclerosis progression.

The performed analysis was built on previous experiences in simulations of atherosclerotic plaque progression within the carotid artery and the obtained results correspond to the previous 
works (Filipovic et al. 2013; Filipovic et al. 2015; Djorovic et al. 2020). Using presented numerical approach, the zone of plaque appearance can be determined, as well as its further propagation. Besides this type of study, previous experimental and numerical studies have examined the influence of haemodynamic and mechanical environments on plaque rupture (Strömberg et al. 2015), which will be the objective of our future studies.

Finally, it should be noted that the current computational model and initial simulation of very complex atherosclerotic process involved some limitations. First of all, the carotid arteries in the human body do not have the same embryological origin and, therefore, might react differently. Second, the average physiological properties and inlet blood velocities were adopted from the literature which does not replicate the patient-specific characteristics. Third, plaque components measured from the medical images should be included in the model. Also, quantification of the real patient-specific plaque progression has to be investigated in order to validate the computational model.
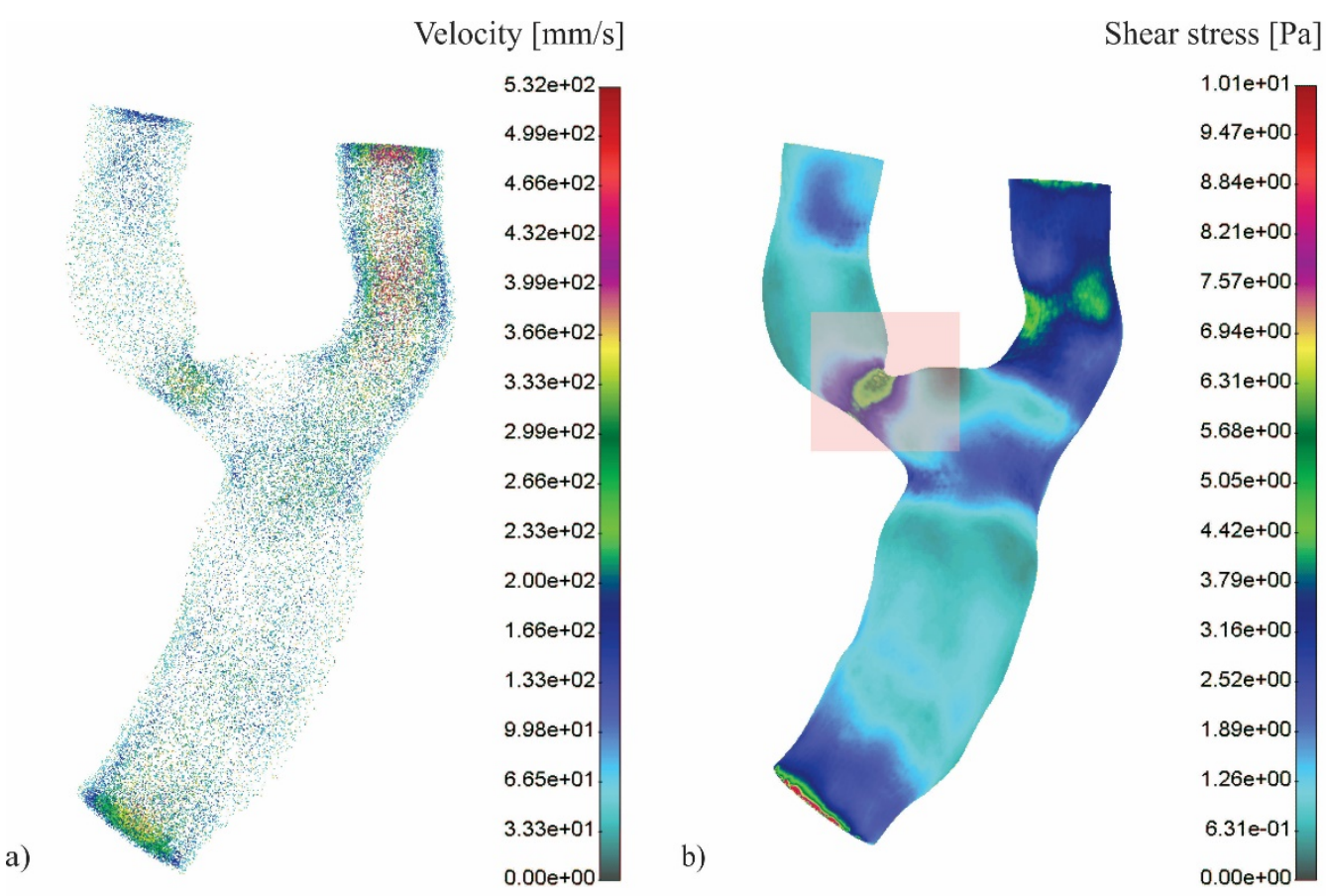

Fig. 4. Follow-up: a) Velocity distribution vector field and b) Shear stress distribution.

\section{Conclusions}

Many researches have been conducted to understand carotid atherosclerosis formation, progression and regression. In this study, the three-dimensional simulation of plaque development was performed in order to investigate the influence of patient-specific geometry, as well as velocity and shear stress distribution on the disease progression. The patient with mild stenosis of ICA was included in the study by using the CT scan images for creation of patientspecific carotid model. During the simulation two time periods were observed (baseline and follow-up). The results of the analysis have shown that zones with decreased blood velocities and lower shear stress values were correlated with the sites of plaque accumulation and progression. Analyzing the follow-up period, low shear stress was still present at the carotid bifurcation which 
together with the presence of the recirculation zone at the carotid bifurcation indicates the possibility for further plaque progression.

Although still affected by several simplifications, the present study can be considered as a further step towards the prediction of disease progression. Therefore, more realistic patientspecific models will be investigated in the next studies, with the aim to constantly improve the computational determination of plaque location and its progression in time, as well as contribute to the daily medical practice.

Acknowledgements: This research is supported by the European Union's Horizon 2020 research and innovation programme under grant agreement No 755320 - TAXINOMISIS. This article reflects only the author's view. The Commission is not responsible for any use that may be made of the information it contains. This paper is also supported by the Ministry of Education, Science and Technological Development of the Republic of Serbia with projects III41007 and OI174028.

\section{References}

Antiga L, Ene-Iordache B, \& Remuzzi A. (2003). Computational geometry for patient-specific reconstruction and meshing of blood vessels from MR and CT angiography. IEEE Trans Med Imaging, 22(5), 674-678.

Bourantas C, et al. (2016). Noninvasive Prediction of Atherosclerotic Progression: The PROSPECT-MSCT Study. JACC Cardiovasc Imaging, 9(8), 1009-11.

Calvez V, Ebde A, Meunier N, \& Raoult A (2008). Mathematical modelling of the atherosclerotic plaque formation. ESAIM Proc, 28, 1-12.

Chaturvedi S, \& Sacco R (2015). How recent data have impacted the treatment of internal carotid artery stenosis. J Am Coll Cardiol, 65(11), 134-43.

Djorovic S, Saveljic I, \& Filipovic N. (2020). Numerical Analysis of Plaque Progression in 3D Patient Specific Model of Carotid Artery. CMBEBIH 2019, IFMBE Proceedings. 73, 337340. Banja Luka, B\&H: Springer, Ch.

Filipovic N, Mijailovic S, Tsuda A, \& Kojic M (2006a). An implicit algorithm within the arbitrary Lagrangian-Eulerian formulation for solving incompressible fluid flow with large boundary motions. Comp Methods Appl Mech Eng, 195, 6347-6361.

Filipovic N, Kojic M, Ivanovic M, Stojanovic B, Otasevic L \& Rankovic V (2006b). MedCFD, Specialized CFD software for simulation of blood flow through arteries. University of Kragujevac, Serbia.

Filipovic N, Saveljic I, Nikolic D, Milosevic Z, Kovacevic P, \& Velicki L (2015). Numerical simulation of blood flow and plaque progression in carotid-carotid bypass patient specific case. Computer Aided Surgery, 20(1), 1-6.

Filipovic N, Teng Z, Radovic M, Saveljic I, Fotiadis D, \& Parodi O (2013). Computer simulation of three dimensional plaque formation and progression in the carotid artery. Med Biol Eng Comput, 51(6), 607-616.

Kamenskiy AV, MacTaggart JN, Pipinos II, Bikhchandani J, \& Dzenis YA (2012). ThreeDimensional Geometry of the Human Carotid Artery. J Biomech Eng, 134(6), 06450210645027.

Kojic M, Filipovic, N, Stojanovic, B, \& Kojic N (2008). Computer Modeling in Bioengineering - Theoretical Background, Examples and Software. Chichester, England: John Wiley and Sons.

Kumar PK, Araki T, Rajan J, Laird JR, Nicolaides A, \& Suri JS (2018). State-of-the-art review on automated lumen and adventitial border delineation and its measurements in carotid ultrasound. Computer Methods and Programs in Biomedicine, 163, 155-168. 
Lee K, Wood N, \& Xu X (2004). Ultrasound image-based computer model of a common carotid artery with a plaque. Med Eng Phys, 26(10), 823-840.

Milašinović, D, Ivanović, M, Tengg-Kobligk, H, Böckler, D, \& Filipović, N (2008). Software Tools for Generating CFD Simulation Models of Blood Flow from CT Images, and for Postprocessing. Journal of the Serbian Society for Computational Mechanics, 2(2), 51-58.

Moussawi, A, Galusinski, C, \& Nguyen C (2014). 3D Reconstruction of Blood Vessels. Engineering with computers, doi: 10.1007/s00366-014-0398-3, 1-16.

Nematollahi, A, Shirani E, Mirzaee I, \& Sadeghi M (2012). Numerical simulation of LDL particles mass transport in human carotid artery under steady state conditions. Scientia Iranica, 19, 519-524.

Parton A, McGilligan V, O'Kane M, Baldrick FR \& Watterson S (2016). Computational modelling of atherosclerosis, Briefings in Bioinformatics, 17(4), 562-575.

Sakellarios A et al. (2017). Prediction of atherosclerotic disease progression using LDL transport modelling: a serial computed tomographic coronary angiographic study. European Heart Journal - Cardiovascular Imaging, 18(1), 11-18.

Salem M et al. (2014). Identification of patients with a histologically unstable carotid plaque using ultrasonic plaque image analysis. European journal of vascular and endovascular surgery, 48(2), 118-125.

Sousa L, Castro C, António C, Sousa F, Santos R, Castro P, \& Azevedo E (2016). Computational simulation of carotid stenosis and flow dynamics based on patient ultrasound data - A new tool for risk assessment and surgical planning. Adv Med Sci, 61(1), 32-39.

Strömberg S, Nordanstig A, Bentzel T, Österberg K, \& Bergström G (2015). Risk of Early Recurrent Stroke in Symptomatic Carotid Stenosis. Eur J Vasc Endovasc Surg, 49(2), 137144.

Tomaso G, Pichardo-Almarza C, Agu O, \& Díaz-Zuccarini V (2015). A Multiscale and PatientSpecific Computational Framework of Atherosclerosis Formation and Progression: A Case Study in the Aorta and Peripheral Arteries. Procedia Computer Science, 51, 1118-1.

Valencia A, \& Baeza F (2009). Numerical simulation of fluid-structure interaction in stenotic arteries considering two layer nonlinear anisotropic structural model. International Communications in Heat and Mass Transfer, 36, 137-142.

Xiaojuan T, Peiyi G, Lina J, Yan L, \& Binbin S (2015). Subject-Specific Fully-Coupled and OneWay Fluid-Structure Interaction Models for Modeling of Carotid Atherosclerotic Plaques in Humans. Med Sci Monit, 21, 279-3290. 